\title{
A NOTE ON DISCONJUGATE DIFFERENTIAL EQUATIONS AND GROWTH ESTIMATES
}

\author{
L. ERBE ${ }^{1}$
}

\begin{abstract}
ABSTRACr. Subfunctions and differential inequality techniques are applied to certain classes of second and third order nonlinear equations to obtain growth estimates on the solutions.
\end{abstract}

1. In several recent papers [1], [2], [18], certain asymptotic properties, disconjugacy, boundedness, and oscillatory behavior have been studied for the equation

$$
L[x](t) \equiv x^{(n)}(t)+\sum_{i=0}^{n-1} a_{i}\left(t, x(t), \ldots, x^{(n-1)}(t)\right) x^{(i)}(t)=0
$$

where $a_{i}\left(t, v_{1}, \ldots, v_{n}\right) i=0, \ldots, n-1$, are continuous on $I \times R^{n}$ and $I \subset R$ is an interval. References [13], [14], [3], [5] contain results obtained for the analogous first order system

$$
x^{\prime}=A(t, x) x+f(t, x)
$$

where $A(t, x)$ is an $n \times n$ matrix valued function defined on $I \times R^{n}$, and $f(t, x)$ and $x$ are $n$-vectors. The results in [1], [2], [18] were obtained by considering the related linear equation

$$
L_{u}[x](t) \equiv x^{(n)}(t)+\sum_{i=0}^{n-1} a_{i}\left(t, u(t), \ldots, u^{(n-1)}(t)\right) x^{(i)}(t)=0
$$

for $u \in C^{(n-1)}(I)$. In particular, a nice application of a fixed-point theorem for multivalued mappings applied to solutions of (3) for $u \in B \subset C^{(n-1)}(I), B$ a bounded closed, convex subset, yields results for equation (1) analogous to those obtained for the linear $n$th order differential equation. In this note we apply subfunction and differential inequality techniques to obtain growth estimates on solutions of (1). For this reason, we restrict attention to the cases $n=2,3$ for which the required theory has been developed (see [16] and the references therein). Thus, we shall consider the equations

$$
x^{\prime \prime}+a_{1}\left(t, x, x^{\prime}\right) x^{\prime}+a_{0}\left(t, x, x^{\prime}\right) x=0
$$

and

$$
x^{\prime \prime \prime}+a_{2}\left(t, x, x^{\prime}, x^{\prime \prime}\right) x^{\prime \prime}+a_{1}\left(t, x, x^{\prime}, x^{\prime \prime}\right) x^{\prime}+a_{0}\left(t, x, x^{\prime}, x^{\prime \prime}\right) x=0 .
$$

Received by the editors September 19, 1980.

1980 Mathematics Subject Classification. Primary 34A10, 34C10, 34C11.

Key words and phrases. Differential inequalities, subfunctions, disconjugacy, asymptotic behaviour.

${ }^{1}$ Research supported by NSERC Grant A-7673. 
In addition to the continuity assumptions already mentioned, we shall assume that for equation (4) the following conditions hold.

$\left(\mathrm{A}_{2}\right)$ If $x_{1}$ and $x_{2}$ are solutions of equation (4) on $\left[t_{1}, t_{2}\right] \subset I$ where $t_{1}<t_{2}$ such that $x_{1}\left(t_{i}\right)=x_{2}\left(t_{i}\right), i=1,2$, then $x_{1}(t) \equiv x_{2}(t)$ on $\left[t_{1}, t_{2}\right]$.

$\left(B_{2}\right)$ All solutions of all initial value problems for (4) extend throughout $I$.

For equation (5) the corresponding assumptions are

$\left(\mathrm{A}_{3}\right)$ If $x_{1}$ and $x_{2}$ are solutions of equation (5) on $\left[t_{1}, t_{2}\right] \subset I$ where $t_{1}<t_{2}$ such that $x_{1}\left(t_{i}\right)=x_{2}\left(t_{i}\right), i=1,2$, and either $x_{1}^{\prime}\left(t_{1}\right)=x_{2}^{\prime}\left(t_{1}\right)$ or $x_{1}^{\prime}\left(t_{2}\right)=x_{2}^{\prime}\left(t_{2}\right)$ then $x_{1}(t)$ $\equiv x_{2}(t)$ on $\left[t_{1}, t_{3}\right]$.

$\left(B_{3}\right)$ All solutions of all initial value problems for (5) extend throughout $I$.

We note that if (4) (or (5)) is linear then condition $\left(A_{2}\right)$ (or $\left(A_{3}\right)$ ) is equivalent to disconjugacy of (4) (or (5)) on $I$ and $\left(B_{2}\right)$ (or $\left(B_{3}\right)$ ) holds trivially. It is known [17] that if $\phi \in C^{2}(I)$ is a lower solution of (4) on $I$; i.e.,

$$
\phi^{\prime \prime}+a_{1}\left(t, \phi, \phi^{\prime}\right) \phi^{\prime}+a_{0}\left(t, \phi, \phi^{\prime}\right) \phi>0
$$

and if $\left(A_{2}\right)$ and $\left(B_{2}\right)$ hold, then $\phi$ is a subfunction with respect to equation (4) on $I$. Similarly, if $\psi \in C^{2}(I)$ is an upper solution on $I$; i.e.,

$$
\psi^{\prime \prime}+a_{1}\left(t, \psi, \psi^{\prime}\right) \psi^{\prime}+a_{0}\left(t, \psi, \psi^{\prime}\right) \psi<0
$$

with $\left(A_{2}\right)$ and $\left(B_{2}\right)$ holding, then $\psi$ is a superfunction with respect to equation (4) on $I$. For the third order case, we need an additional assumption which is:

$\left(\mathrm{C}_{3}\right)$ Either $f\left(t, x, x^{\prime}, x^{\prime \prime}\right) \equiv-\Sigma_{i=0}^{2} a_{i}\left(t, x, x^{\prime}, x^{\prime \prime}\right) x^{(i)}$ in (5) satisfies a Lipschitz condition with respect to $x, x^{\prime}$, and $x^{\prime \prime}$ on compact subsets of $I \times R^{3}$ or else $\phi, \psi \in C^{3}(I)$ are strict lower and upper solutions, respectively, for (5) on $I$.

We then have the result [8], [11]. If $\phi \in C^{3}(I)$ is a lower solution for (5) (i.e., $\left.\phi^{\prime \prime \prime}+\sum_{i=0}^{2} a_{i}\left(t, \phi, \phi^{\prime}, \phi^{\prime \prime}\right) \phi^{(i)} \geqslant 0\right)$ and if $\left(\mathrm{A}_{3}\right),\left(\mathrm{B}_{3}\right)$ and $\left(\mathrm{C}_{3}\right)$ hold, then $\phi$ is a subfunction with respect to (5) on $I$. Similarly, if $\psi \in C^{3}(I)$ is an upper solution for (5) (i.e., $\left.\psi^{\prime \prime \prime}+\sum_{i=0}^{2} a_{i}\left(t, \psi, \psi^{\prime}, \psi^{\prime \prime}\right) \psi^{(i)}<0\right)$ and if $\left(\mathrm{A}_{3}\right),\left(\mathrm{B}_{3}\right),\left(\mathrm{C}_{3}\right)$ hold, then $\psi$ is a superfunction with respect to (5) on $I$.

We note also that for equation (4) assumptions $\left(A_{2}\right)$ and $\left(B_{2}\right)$ imply that all two point boundary value problems on $I$ do have solutions [12]. That is, for any $t_{1}$, $t_{2} \in I, t_{1}<t_{2}$, and any $c, d \in R$ there is a (unique) solution to (4) satisfying $y\left(t_{1}\right)=c, y\left(t_{2}\right)=d$. Similarly, assumptions $\left(\mathrm{A}_{3}\right)$ and $\left(\mathrm{B}_{3}\right)$ imply that all two point and three point boundary value problems for equation (5) have unique solutions [8, Theorem 2, p. 435]. As in [16] we shall assume that lower solutions are greater than or equal to upper solutions which is opposite to that usually found in papers dealing with second and third order equations.

2. The first two results are restatements or slight extensions of results of Schrader [16] and, since they do not depend on the particular form of the equation, are valid for the more general equations

$$
x^{\prime \prime}=f\left(t, x, x^{\prime}\right) \text { and } \quad x^{\prime \prime \prime}=f\left(t, x, x^{\prime}, x^{\prime \prime}\right) .
$$

TheOREM 2.1. (a) Let $I \subset R$ be an interval and assume that $\left(\mathrm{A}_{2}\right)$ and $\left(\mathrm{B}_{2}\right)$ hold for equation (4). Let $\phi, \psi \in C^{2}(I)$ be lower and upper solutions, respectively, of (4) on I 
with $\psi(t)<\phi(t)$ on I. Then there is a solution $x$ of (4) on I with $\psi(t)<x(t)<\phi(t)$ on $I$.

(b) Further, if $I=[a, b]$ is compact and $\psi(t)<\phi(t)$ on $[a, b]$ then there exist two distinct solutions $x, y$ of (4) with $\psi(t)<x(t)<y(t)<\phi(t)$ on $[a, b]$.

Proof. Part (a) is Theorem 2.2 of [16]. So suppose $I=[a, b]$ and $\psi(t)<\phi(t)$ on $[a, b]$. It suffices to show that there exists a solution $x$ of (4) with $\psi(t)<x(t)<\phi(t)$ on $[a, b]$ for then by an analogous argument there will exist a solution $y$ of (4) with $x(t)<y(t) \leqslant \phi(t)$ on $[a, b]$. Following Schrader [16, Theorem 2.2, p. 207] for each $\lambda \in[a, b]$ let $\left\{v_{n}\right\}$ and $\left\{y_{n}\right\}$ be sequences of solutions of $(4)$ on $[a, b]$ satisfying the respective initial conditions

$$
v_{n}(\lambda)=\psi(\lambda), \quad v_{n}^{\prime}(\lambda)=\psi^{\prime}(\lambda)+1 / n
$$

and

$$
y_{n}(\lambda)=\psi(\lambda), \quad y_{n}^{\prime}(\lambda)=\psi^{\prime}(\lambda)-1 / n
$$

It follows as in [16] that there exist solutions $v$ and $y$ of (4) on $[a, b]$ which are limits of subsequences of $\left\{v_{n}\right\}$ and $\left\{y_{n}\right\}$, respectively, with $v(\lambda)=\psi(\lambda), v^{\prime}(\lambda)=$ $\psi^{\prime}(\lambda), v(t) \geqslant \psi(t)$ for $\lambda \leqslant t \leqslant b$ and $y(\lambda)=\psi(\lambda), y^{\prime}(\lambda)=\psi^{\prime}(\lambda), y(t) \geqslant \psi(t)$ on $a<t<\lambda$. Thus the solution $x_{\lambda}$ of (4) defined by $x_{\lambda}(t)=y(t)$ for $a<t<\lambda$ and $x_{\lambda}(t)=v(t)$ for $\lambda \leqslant t \leqslant b$ cannot (by [17, Theorem 1, p. 1007]) be equal to $\phi(t)$ at two points $t_{1}(\lambda), t_{2}(\lambda) \in[a, b]$ with $t_{1}(\lambda)<\lambda<t_{2}(\lambda)$. We claim that there exists $\lambda_{0} \in[a, b]$ such that $\psi(t) \leqslant x_{\lambda_{0}}(t)<\phi(t)$ on $[a, b]$. If not, then for each $\lambda \in[a, b]$ either $t_{1}(\lambda)<\lambda$ exists with $x_{\lambda}\left(t_{1}(\lambda)\right)=\phi\left(t_{1}(\lambda)\right)$ or else $t_{2}(\lambda)>\lambda$ exists with $x_{\lambda}\left(t_{2}(\lambda)\right)$ $=\phi\left(t_{2}(\lambda)\right)$. Let $T_{1}=\left\{\lambda \in[a, b]: t_{1}(\lambda)\right.$ exists $\}$ and $T_{2}=\left\{\lambda \in[a, b]: t_{2}(\lambda)\right.$ exists $\}$. Since $t_{1}(b) \in T_{1}$ and $t_{2}(a) \in T_{2}$ neither $T_{1}$ nor $T_{2}$ is empty and $T_{1} \cap T_{2}=\varnothing$, $T_{1} \cup T_{2}=[a, b]$. Suppose $\left\{\lambda_{n}\right\} \subset T_{1}$ is a sequence and $\lambda_{n} \rightarrow \hat{\lambda}$. Then $\left\{t_{1}\left(\lambda_{n}\right)\right\} \subset$ $[a, b]$ and there exists $x_{n}=x_{\lambda_{n}}$ a sequence of solutions of (4) with $x_{n}\left(t_{1}\left(\lambda_{n}\right)\right)=$ $\phi\left(t_{1}\left(\lambda_{n}\right)\right)$. There exists (by [7, Theorem 3, p. 14]) a subsequence of $\left\{x_{n}\right\}$ and $\left\{t_{1}\left(\lambda_{n}\right)\right\}$ which we again label $\left\{x_{n}\right\}$ and $\left\{t_{1}\left(\lambda_{n}\right)\right\}$ such that $t_{1}\left(\lambda_{n}\right) \rightarrow \hat{t}$ and $x_{n}$ converges (uniformly on $[a, b]$ ) to a solution $\hat{x}$ of (4). Thus $\hat{x}(\hat{t})=\lim _{n \rightarrow \infty} x_{n}\left(t_{1}\left(\lambda_{n}\right)\right)=$ $\lim _{n \rightarrow \infty} \phi\left(t_{1}\left(\lambda_{n}\right)\right)=\phi(\hat{t})$ so that $\hat{t}=\hat{t_{1}}(\hat{\lambda})$ and $\hat{x}=\hat{\lambda_{\lambda}}$. Hence, $T_{1}$ is closed so $T_{2}$ is open. Similarly, $T_{2}$ is closed. This contradiction shows that there exists $\lambda_{0} \in$ $[a, b] \backslash\left(T_{1} \cup T_{2}\right)$ and therefore $\psi(t)<x_{\lambda_{0}}(t)<\phi(t)$ on $[a, b]$, as claimed. Now by an analogous argument with $\phi$ replacing $\psi$ in conditions (8) and (9) we conclude the existence of a solution $y_{\lambda_{1}}$ of (4) with $x_{\lambda_{0}}(t)<y_{\lambda_{1}}(t)<\phi(t)$ on $[a, b]$. This completes the proof.

For completeness we include the following

THEOREM 2.2 (SCHRADER [16]). Let $I \subset R$ be an interval, $\alpha \in I^{0}$ and assume that $\left(\mathrm{A}_{3}\right),\left(\mathrm{B}_{3}\right)$ hold. Let $\phi, \psi \in C^{3}(I)$ be lower and upper solutions, respectively, for (5) on $I$ with $\psi(t) \geqslant \phi(t)$ on $(-\infty, \alpha] \cap I$ and $\psi(t)<\phi(t)$ on $[\alpha,+\infty) \cap I$. If $\left(\mathrm{C}_{3}\right)$ holds then there is a solution $x$ of (5) on $I$ with $\psi(t)>x(t)>\phi(t)$ on $(-\infty, \alpha] \cap I$ and $\psi(t)<x(t)<\phi(t)$ on $[\alpha,+\infty) \cap I$.

Proof. See Theorem 3.6 of [16]. 
COROllary 2.3. Let $I \subset R$ be an interval let $\left(\mathrm{A}_{3}\right),\left(\mathrm{B}_{3}\right)$ hold, and for each $n>1$ assume that $\phi_{n}, \psi_{n}$ are lower and upper solutions, respectively of $(5)$ on $I$ with $\left(\mathrm{C}_{3}\right)$ holding and with $\psi_{n}(t) \geqslant \phi_{n}(t)$ for $t \in\left(-\infty, \alpha_{n}\right) \cap I$ and $\psi_{n}(t)<\phi_{n}(t)$ for $t \in$ $\left[\alpha_{n},+\infty\right) \cap I$ where $\alpha_{n} \in I^{0}$. Assume further that $\phi_{n}, \psi_{n}$ are uniformly bounded on each compact subinterval of I and that $\lim _{n \rightarrow \infty} \phi_{n}(t) \equiv \hat{\phi}(t)$ and $\lim _{n \rightarrow \infty} \psi_{n}(t) \equiv \hat{\psi}(t)$ exist uniformly on compact subintervals of $I$ and that $\lim _{n \rightarrow \infty} \alpha_{n}=\hat{\alpha}(\hat{\alpha}= \pm \infty$ is allowed). Then there exists a solution $x$ of (5) such that $\hat{\phi}<x<\hat{\psi}$ on $(-\infty, \hat{\alpha}] \cap I$ and $\hat{\psi}<x \leqslant \hat{\phi}$ on $[\hat{\alpha}, \infty) \cap I$.

Proof. For each $n \geqslant 1$ let $x_{n}$ satisfy the conclusion of Theorem 2.2. Then since $\left\{x_{n}\right\}$ is a uniformly bounded sequence on each compact subinterval of $I$ it follows by [12, Theorem 1] that there exists a subsequence of $\left\{x_{n}\right\}$ and a solution $x$ of (5) such that $\lim x_{n}^{(i)}(t)=x^{(i)}(t), i=0,1,2$, uniformly on compact subintervals. If $\hat{\alpha}=+\infty$ then $\hat{\phi} \leqslant x \leqslant \hat{\psi}$ on $I$ and if $\hat{\alpha}=-\infty$ then $\hat{\psi}<x<\hat{\phi}$ on $I$. If $\hat{\alpha} \in$ $(-\infty,+\infty)$, then since $\phi_{n} \leqslant x \leqslant \psi_{n}$ on $\left(-\infty, \alpha_{n}\right] \cap I$ and $\psi_{n}<x_{n}<\phi_{n}$ on $\left[\alpha_{n},+\infty\right) \cap I$, the conclusion follows.

3. Examples and applications. We shall now illustrate the applicability of the above subfunction-differential inequality technique to equations of the form (4) and (5). In particular, the results of [1], [2] do not apply in the following examples.

EXAMPLE 3.1. Let equation (4) satisfy $\left(\mathrm{A}_{2}\right)$ and $\left(\mathrm{B}_{2}\right)$ on $I$ and assume there exist constants $\beta<\alpha$ with $a_{0}(t, \beta, 0) \beta \leqslant 0 \leqslant a_{0}(t, \alpha, 0) \alpha$. Then there exists a solution $x(t)$ of (4) with $\beta \leqslant x(t) \leqslant \alpha$ on $I$.

EXAMPLE 3.2. Let equation (4) satisfy $\left(\mathrm{A}_{2}\right)$ and $\left(\mathrm{B}_{2}\right)$ and assume there exists $\lambda>0$ such that $\lambda^{2}+a_{1}\left(t, x, x^{\prime}\right) \lambda+a_{0}\left(t, x, x^{\prime}\right) \geqslant 0$ for all $|x|+\left|x^{\prime}\right|<+\infty$ and $t \in I$ $=[a, b]$. Then there exist solutions $x(t), y(t)$ of (4) such that $0<x(t)<y(t)<e^{\lambda_{t}}$ on $[a, b]$. (Here $\psi(t) \equiv 0$ and $\phi(t) \equiv e^{\lambda t}$ are upper and lower solutions, respectively, so Theorem $2.1 \mathrm{~b}$ yields the result.)

EXAMPLE 3.3. Let there exist $0<\mu<1<\nu$ such that $-\nu(\nu-1)<t^{2} p(t)<$ $\mu(1-\mu)$ on $[a,+\infty), a>0$. The Sturm Comparison Theorem [19] shows then that the linear equation

$$
x^{\prime \prime}+p(t) x=0
$$

is disconjugate on $[a,+\infty$ ) (by comparison with the disconjugate Euler equation $x^{\prime \prime}+\frac{1}{4} t^{-2} x=0$ ). Therefore from Theorem 2.1 (with $\psi(t) \equiv t^{\mu}<t^{\nu} \equiv \phi(t)$ ) we conclude the existence of a solution $x(t)$ of (10) with $t^{\mu}<x(t)<t^{\nu}$. Further, from results of [9, Theorem 7.4] there also exists a solution $y(t)$ of $(10)$ with $0<y(t)<t^{\mu}$ on $[a,+\infty)$.

We conclude with several examples for the case $n=3$.

EXAMPLE 3.4. Consider the third order linear equation

$$
x^{\prime \prime \prime}+p(t) x=0
$$

and assume that $\left|t^{3} p(t)\right| \leqslant 2 / 3 \sqrt{3}$ for $t \geqslant a$ where $0<a<1$. From [19, p. 163] it follows (by comparison with the disconjugate Euler equations $x^{\prime \prime \prime} \pm 2 t^{-3} x / 3 \sqrt{3}=$ 0 on $[a,+\infty), a>0)$ that $(11)$ is disconjugate on $[a,+\infty)$ so that $\left(A_{3}\right),\left(B_{3}\right),\left(C_{3}\right)$ 
hold. With $\mu=1+\sqrt{3} / 3$ and $\nu>2$ the unique solution of $\nu(\nu-1)(\nu-2)=$ $2 / 3 \sqrt{3}$ it follows that $\phi(t) \equiv t^{\nu}$ and $\psi(t) \equiv t^{\nu}$ are lower and upper solutions on $[a,+\infty)$. Therefore Theorem 2.2 implies the existence of a solution $x(t)$ of (11) satisfying $t^{\mu} \leqslant x(t) \leqslant t^{\nu}$ on $[1,+\infty)$ and $t^{\nu}<x(t)<t^{\mu}$ on $[a, 1]$. We note that the results of [2] (see also [10]) concerning the separation of the roots of the characteristic equation $\lambda^{3}+p(t)=0$ by constants is never fulfilled for an equation of the form (11) so that those techniques are not applicable here.

EXAMPLE 3.5. Consider equation

$$
x^{\prime \prime \prime}+a_{2}\left(t, x, x^{\prime}\right) x^{\prime \prime}+a_{1}\left(t, x, x^{\prime}\right) x^{\prime}+a_{0}\left(t, x, x^{\prime}\right) x=0
$$

and suppose conditions $\left(\mathrm{A}_{3}\right),\left(\mathrm{B}_{3}\right)$ hold on $I=[a, b]$ and let there exist constants $\rho_{1}<\rho_{2}$ with

$$
\rho_{1}^{3}+a_{2}\left(t, x, x^{\prime}\right) \rho_{1}^{2}+a_{1}\left(t, x, x^{\prime}\right) \rho_{1}+a_{0}\left(t, x, x^{\prime}\right)<0
$$

and

$$
\rho_{2}^{3}+a_{2}\left(t, x, x^{\prime}\right) \rho_{2}^{2}+a_{1}\left(t, x, x^{\prime}\right) \rho_{2}+a_{0}\left(t, x, x^{\prime}\right)>0
$$

for all $|x|+\left|x^{\prime}\right|<+\infty$. Then it follows that for any $a<t_{0}<b, \psi_{t_{0}}(t) \equiv e^{\left(t-t_{0}\right) \rho_{1}}$ and $\phi_{t_{0}}(t) \equiv e^{\left(t-t_{0}\right) \rho_{2}}$ are upper and lower solutions for $(5)$ on $[a, b]$. Thus by Theorem 2.2 (or Corollary 2.3) there exists a solution $x\left(t ; t_{0}\right)$ of $(5)$ with $\phi_{t_{0}}(t) \leqslant$ $x\left(t ; t_{0}\right) \leqslant \psi_{t_{0}}(t)$ on $\left[a, t_{0}\right]$ and $\psi_{t_{0}}(t) \leqslant x\left(t ; t_{0}\right)<\phi_{t_{0}}(t)$ on $\left[t_{0}, b\right]$. This result may not be concluded from the results of [1], [2]. We note that if $a_{i}\left(t, x, x^{\prime}\right)$ are bounded on $[a, b] \times R^{2}$ then (12) and (13) can always be satisfied for sufficiently large negative $\rho_{1}$ and large positive $\rho_{2}$.

\section{REFERENCES}

1. G. Anichini and J. D. Schuur, Using a fixed point theorem to describe the asymptotic behavior of solutions of a class of nonlinear ordinary differential equations, (Proc. Conf. Equadiff 78, Florence, Italy, March, 1978), pp. 245-256.

2. Acad. Polon. Sci. Sér. Sci. Math. Astronom. Phys. 26 (1978), 787-790.

3. R. Conti, Sistemi differenziali ordinari con condizioni lineari, Ann. Mat. Pura. Appl. 46 (1958), 109-130.

4. W. A. Coppel, Disconjugacy, Lecture Notes in Math., vol. 220, Springer-Verlag, Berlin and New York, 1971.

5. C. Corduneanu, Sur les systèmes différentiels de la forme $y^{\prime}=A(x, y) y+b(x, y)$, An. Şti. Univ. “Al. I. Cuza” Iaşi Secţ. I. Mat. (N.S.) 4 (1958), 45-52.

6. P. Hartman, Disconjugacy and Wronskians, Japan-U.S. Seminar on Ordinary Differential Equations and Functional Equations (M. Urabe, Ed.), Lecture Notes in Math., vol. 243, Springer-Verlag, Berlin and New York, 1971, pp. 208-218.

7. , Ordinary differential equations, Wiley, New York, 1964.

8. L. K. Jackson, Existence and uniqueness of solutions of boundary value problems for third order differential equations, J. Differential Equations 13 (1973), 432-437.

9. $307-363$.

10. Disconjugacy conditions for linear third order differential equations, J. Differential Equations 4 (1968), 369-372.

11. L. Jackson and K. Schrader, Subfunctions and third order differential inequalities, J. Differential Equations 8 (1970), 180-194.

12. Existence and uniqueness of solutions of boundary value problems for third order differential equations, J. Differential Equations 9 (1971), 46-54. 
13. A. G. Kartsatos, Nonzero solutions to boundary value problems for nonlinear systems, Pacific J. Math. 5 (1974), 425-433.

14. _ Bounded solutions to perturbed nonlinear systems and asymptotic relationships, J. Reine Angew. Math. 273 (1975), 170-177.

15. A. Ju. Levin, Nonoscillation of solutions of the equation $x^{(n)}+p_{1}(t) x^{(n-1)}+\cdots+p_{n}(t) x=0$, Russian Math. Surveys 24 (1969), 43-49.

16. K. W. Schrader, Differential inequalities for second and third order equations, J. Differential Equations 25 (1977), 203-215.

17., A note on second order differential inequalities, Proc. Amer. Math. Soc. 19 (1968), 1007-1012.

18. J. D. Schuur, A class of nonlinear ordinary differential equations which inherit linear-like asymptotic behavior, Nonlinear Analysis, T.M.A. 3 (1979), 81-86.

19. C. A. Swanson, Comparison and ascillation theory of linear differential equations, Academic Press, New York, 1968.

Department of Mathematics, University of Alberta, Edmonton, Alberta, Canada T6G 2 H1 